\title{
Comparison on postural control between abdominal draw-in maneuver and abdominal expansion maneuver in persons with stroke
}

\author{
Ho-Suk Choi ${ }^{a}$, Yu-Jin Shim ${ }^{a, b}$, Won-Seob Shin ${ }^{a, c}$ \\ ${ }^{a}$ Department of Physical Therapy, Graduate School of Daejeon University, Daejeon, Republic of Korea \\ ${ }^{\mathrm{b}}$ Department of Hospital Management, International University of Korea, Jinju, Republic of Korea \\ ${ }^{\mathrm{c} D}$ epartment of Physical Therapy, College of Health and Medical Science, Daejeon University, Daejeon, Republic of Korea
}

Objective: The effect of abdominal expansion maneuver (AEM) and abdominal draw-in maneuver (ADIM) on postural control in an unsupported position in stroke patients.

Design: Randomized controlled trial.

Methods: A total of 36 persons with hemiplegic stroke participated in this study. The subjects were randomly divided into an AEM experimental group $(n=12)$, an experimental ADIM group $(n=12)$, and a control group $(n=12)$. We collected the general characteristics of all subjects and the pre-test results before the intervention and after 4 weeks of the intervention. The trunk stabilization training of the ADIM and AEM group were performed 15 minutes a day, 3 times a week for 4 weeks, and general physical therapy was performed 2 times a day, 30 minutes per session, 5 times a week for all three groups. The control group received joint mobilizations, muscle strengthening, endurance strengthening, and gait exercises along with treatment of the central nervous system, such as neuro-developmental treatment, mat, and gait training. The AEM is an inspiratory phase of tidal breathing expanding the lateral lower ribcage in a lateral direction with minimal superior movements of the chest. Then the lower abdomen expands and the navel moves in an anterior-caudal direction. The ADIM is a repeated contraction and relaxation of the anal sphincter during inspiration. The navel pulls the lower abdomen to the direction of the spine without the movement of the trunk and pelvis.

Results: Before and after the interventions, medial-lateral axis movement distance, anterior-posterior axis movement distance, sway mean velocity, and sway area $95 \%$ was a statistically significant change in all three groups $(p<0.05)$. The post-hoc test showed a significant improvement in medial-lateral axis movement distance, anterior-posterior axis movement distance, sway mean velocity, and sway area in the AEM group compared with the control group, and in the ADIM group compared with the control group $(p<0.05)$.

Conclusions: In conclusion, both AEM training and ADIM training are necessary interventions to maintain the independent sitting position according to the characteristics of the patient.

Key Words: Postural balance, Respiratory therapy, Stroke

\section{Introduction}

Stroke is the major cause of chronic disability in adults and $50 \%$ of them could regain functional independence of $50 \%-70 \%$ within 6 months [1]. Stroke is subject to serious limitations in physical activity with movement disorders such as functional and sensory functions [2]. Resulting loss of trunk control can cause malalignment of the body with decreasing motor function of the affected upper and lower extremities. It is involved in such as gait disturbances, balance disorders, and breathing disorders $[3,4]$. Loss of selective trunk movement of the stroke patient means reducing

Received: 19 August, 2016 Revised: 7 September, 2016 Accepted: 7 September, 2016

Corresponding author: Won-Seob Shin

Department of Physical Therapy, Daejeon University, 62 Daehak-ro, Dong-gu, Daejeon 34520, Republic of Korea

Tel: 82-42-280-2294 Fax: 82-42-280-2295 E-mail: shinws@dju.kr

(c) This is an Open-Access article distributed under the terms of the Creative Commons Attribution Non-Commercial License (http://creativecommons.org/licens es/by-nc/4.0) which permits unrestricted non-commercial use, distribution, and reproduction in any medium, provided the original work is properly cited.

Copyright $@ 2016$ Korean Academy of Physical Therapy Rehabilitation Science 
the stability to maintain posture, and such damage is related to the muscles directly to maintain the posture and there is a relationship with which is associated with the respiratory muscles [4]. The control function of the trunk plays an important role in maintaining and movement of the standing posture, body stabilization for activities of daily living, and the recovery of balance and stability. In addition, it is necessary in order to improve the quality of life and levels of physical function levels [5].

The weakness of the trunk muscles to the stroke patient is closely related to life support [6], reducing of the necessary motion control to maintain the cooperative action, degrade the movement of the rib cage affected to the normal posture control of the trunk and the respiratory muscles [7]. The damaged trunk muscles lead to dysfunction of the trunk postures, weakening the breathing function [8]. In general, the deep abdominal muscles contributing to the stability of the trunk in cooperation with the deep multifidi [9]. Decreased postural stability of the trunk interfere the recovery of the overall functional performance of stroke patients. The ability of unsupported sitting is one of the highest predictors for functional outcome in stroke patients $[10,11]$. Because the postural stability of the trunk in unsupported, sitting is required for resistance to the clerical up movement (rising from a seated position), standing posture, walking, lean or tilt movement, and stretching arms and sway [12].

The abdominal expansion maneuver (AEM) does not extend to the rib cage during inspiration, if kept while the lower abdomen is expanded, the navel is stretched out in a caudal-anterior direction. It could increase the simultaneous contraction of the diaphragm and deep spinal stabilizer muscles and intra-abdominal pressure (IAP). This is required to provide forward stability of the lumbopelvis position $[13,14]$. Abdominal draw-in maneuver (ADIM) is commonly used in a clinical setting and is a technique that stabilizes the trunk with faster selective contraction of the transverse abdominis, rectus abdominis muscle, obliqus abdominus externi, and obliqus abdominus interni than other superficial muscles. Neuromuscular re-education through the transverse abdominis and obliqus abdominus interni is a method widely used to improve the stability of the lumbar spine $[15,16]$. A person with a visual feedback using a pressure biofeedback unit or ultrasound image in order to accurately perform the ADIM is how to determine the contraction of the abdominal muscles have been used.

A study of sitting balance generally uses the global clinical measure by using the trunk control test and the trunk im- pairment scale with basic motion coupled to the associated trunk control and the postural assessment scale for stroke patients, and so on [17-19].

The force platform has been used for quantitative evaluation of sitting-balance [20]. Quantitative evaluation of balance control of stroke patients is possible with the use of the force platform for the sway area, maximum displacements or average velocity while maintaining unsupported sitting [21].

In a prior studies of stroke patients, clinical balance ability, upper extremity function, pulmonary function by applying the trunk stabilization exercises such as ADIM, AEM and comparative studies of the normal person have been studied [14]. However, studies with ADIM and AEM training interventions being implemented in trunk stabilization training with a quantitative evaluation of the impact on postural stability in unsupported sitting is not enough. Therefore, in this study, in order to understand the impact of unsupported sitting postural control when applying the AEM and ADIM to stroke patients, we performed this study.

\section{Methods}

\section{Subjects}

The subjects of this study were 36 people in hemiplegic stroke patients receiving inpatient care at Daejeon Hospital located in Daejeon. The purpose and procedures of the study were fully informed to the subjects who participated and all the subjects voluntarily signed a consent to participate. The subjects were randomly divided into the AEM experimental group $(\mathrm{n}=12)$, the ADIM experimental group $(\mathrm{n}=12)$, and a control group $(n=12)$ to receive general neurological rehabilitation physiotherapy. The selection criteria of the subjects were persons with more than six months after being diagnosed with stroke, and persons who do not have cognitive impairment and obtained more than 24 points in the Korean version of the mini-mental status examination.

The exclusion criteria of subjects were persons with other neurological problems or orthopedic injury other than the stroke that could affect the result of this study, persons with history of particular pulmonary disease before stroke, and persons with associated injuries such as congenital deformity of thoracic spine or rib fractures. This study was conducted with the approval of the institutional review board of Daejeon University (IRB no. 1040647-201412HR-012-03). 


\section{Procedure}

In this study, the subjects were randomly divided into three groups to study the effect of stabilizing the trunk and pulmonary function in accordance with the kind of breathing method. Subjects were randomly selected by drawing out of a box pieces of paper labelled 1, 2, or 3. Subjects all agreed to participate after explanation of the purpose and method of this study. Subjects who picked group 1 were assigned to the AEM experimental group, the subjects picked group 2 were assigned to the ADIM experimental group, and the subjects picked group 3 were assigned to the control group. The data in the experiment was collected to investigate the general characteristics and pre-test and perform the post-test after intervention of four weeks and analyzed the measured value of each. The trunk stabilization training of ADIM group and AEM group were performed 15 minutes a day, 3 times a week, and for 4 weeks, and the general physical therapy was performed 2 times a day, 30 minutes per session, 5 times a week to all three groups.

\section{AEM}

The AEM experiment group was instructed with the following with AEM, lower ribs moves to the side and the ster- num toward the abdomen, but the abdomen does not rise in accordance with breathing and the navel does not move in the cephalic direction during inspiration. Expansion occurred not only to the inside of the abdomen, but in all directions (side and rear). Also, the 5th-8th rib area no longer expanded during inhalation (Figure 1). The abdomen was so stretched out with the navel reaching out anterior inferiorly (direction of the pubic symphysis). The upper abdomen did not expand lower than the abdomen, and it was done to avoid movements, such as forward tilt of the pelvis. Subjects were instructed to keep breathing in this state and to keep the posture for 10 seconds. After repeating the AEM 10 times, a 3-minute break was given and an application of 3 sets of AEM for 15 minutes was administered in total [14].

\section{ADIM}

The ADIM experiment group was instructed with the following. ADIM is repeated contraction and relaxation of the anal sphincter during inspiration with the navel pulling the lower abdomen in the direction of the spine without the movement of the trunk and pelvis. This breathing position is lightly held for 10 seconds (Figure 2). The posture to place the pad on the abdomen of the Pressure Bio-Feedback unit (Chattanooga Medical Supply, Inc., Chattanooga, TN,

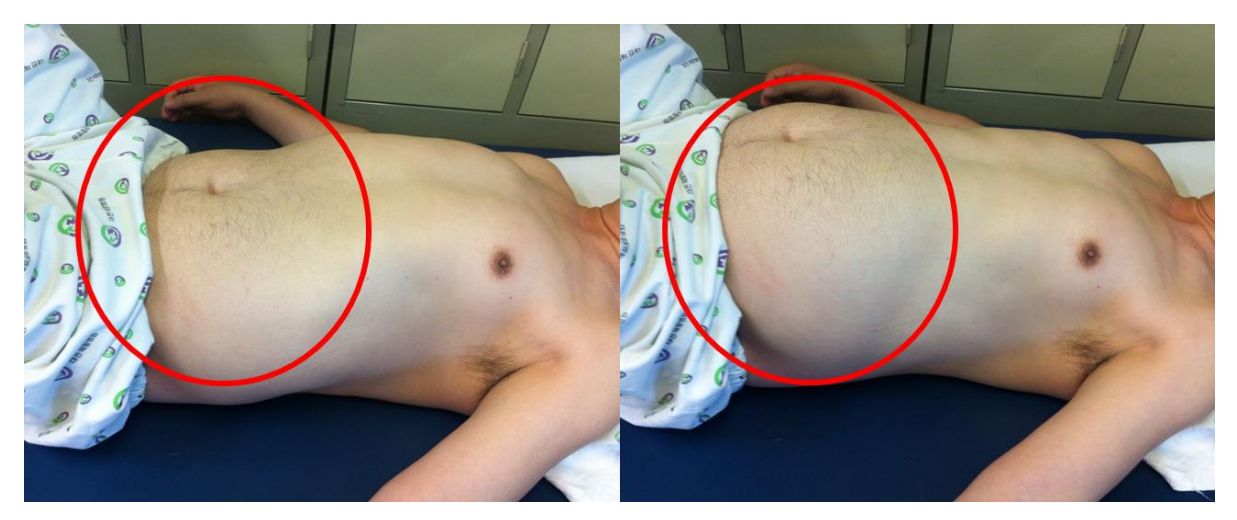

Figure 1. Abdominal expansion maneuver.
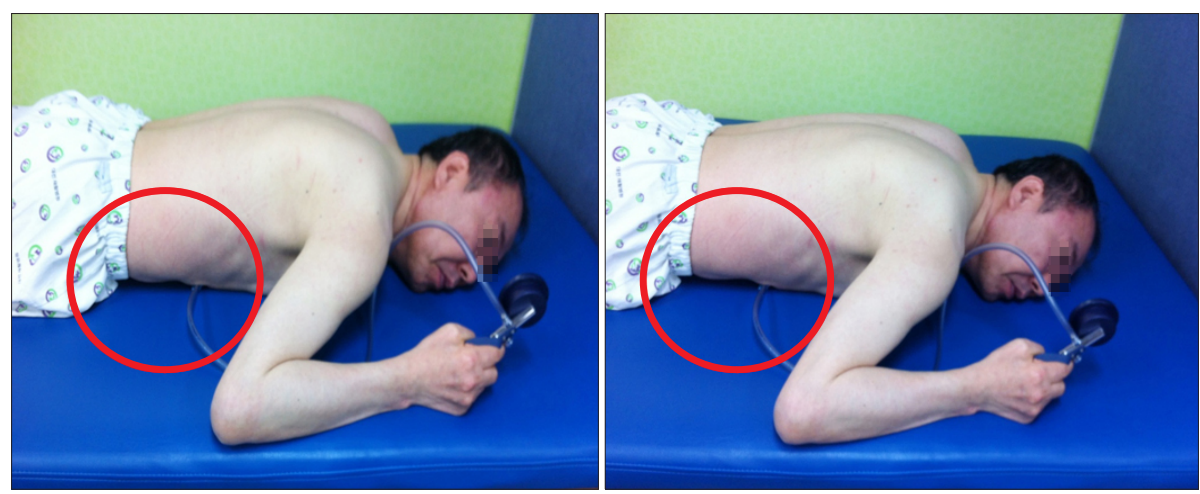

Figure 2. Abdominal draw-in maneuver. 
USA) was in the prone position, the gauge was used to indicate the pressure to $70 \mathrm{mmHg}$. The subjects were trained pressures that are connected to the pressure bio-feedback device to pull the abdomen towards the spine so that the degree of reduction was about $6-10 \mathrm{mmHg}$. In this state, the subjects held the breathing position and maintained the posture for 10 seconds. After 10 repetitions, a 3 minute break was given and an application of 3 sets of ADIM for 15 minutes was administered in total [22].

\section{Control group}

The control group received physical therapy joint mobilizations, muscle strengthening, endurance strengthening exercises, and gait exercises along with treatment of the central nervous system, such as neuro-development treatment, mat and gait training.

\section{Outcome measures}

We used a Wii Balance Board (WBB; Nintendo, Kyoto, Japan) to evaluate the balance ability of sitting postures in stroke patients. The size of the WBB is $45.0 \times 26.5 \mathrm{~cm}$, and with special built chair with the legs of chairs in each corner of the WBB, the center of pressure (COP) was measured in sitting. The WBB provides COP information which was collected continuously through the road-cells in the four corners of the WBB, and the data was collected through a computer device connected via Bluetooth. Sampling rate of the collected data was controlled by the associated software. In this study, the COP movement distance of medial-lateral (ML) and anterior-posterior (AP) axes (cm), sway mean velocity $(\mathrm{cm} / \mathrm{s})$ and sway area $95 \%\left(\mathrm{~cm}^{2}\right)$ were all collected and measured, and all data were sampled is at $100 \mathrm{~Hz}$, and performed at $10 \mathrm{~Hz}$ low-pass filter. COP measurements was 30 seconds when measured once measured, the collected data was analyzed with the Valencia program (Mintosys, Seoul,
Korea), after measuring the average value of 3 trials [23].

\section{Data and statistical analysis}

We used PASW Statistics ver. 18.0 (IBM Co., Armonk, NY, USA) for the statistical processing of the data. General characteristics of the subjects were presented as mean and standard deviation (SD) using descriptive statistics. Chisquare test was used to investigate the change before and after in each group was used for paired t-test. One-way analysis of variance (one-way ANOVA) was conducted to investigate the difference among the groups of the movement distance of ML and AP axes, sway velocity and sway area at $95 \%$ according to the type of breathing exercise. The Scheffe test was used for post-hoc and the level of significance was set to $\alpha=0.05$.

\section{Results}

Thirty-six subjects participated in this study, and were randomly divided to AEM experimental group ( $\mathrm{n}=12)$, ADIM experimental group $(n=12)$, and a control group $(n=12)$. There was no significant difference $(p>0.05)$ among the three groups in gender, age, height, weight, paralyzed side, pathogenesis, and onset period. General characteristics of the subjects in this study are as follows (Table 1).

Before and after the interventions, there was a significant change $(p<0.05)$ in all three groups in the ML axis movement distance while the post-hoc test showed no significant difference among the three groups. There was a significant change $(p<0.05)$ in all three groups in the AP axis movement distance after the interventions, while the post-hoc test showed no significant difference among the three groups. For sway mean velocity, there was a significant change in all three groups before and after the interventions $(p<0.05)$. In post-hoc test result, there was a significant difference in the

Table 1. The general characteristics of all subjects

$(\mathrm{N}=36)$

\begin{tabular}{lcccc}
\hline \multicolumn{1}{c}{ Characteristic } & ADIM group $(\mathrm{n}=12)$ & AEM group $(\mathrm{n}=12)$ & Control group $(\mathrm{n}=12)$ & $\chi^{2} / \mathrm{F}$ \\
\hline Gender (male/female) & $5 / 7$ & $5 / 7$ & $8 / 4$ & 0.368 \\
Age (y) & $61.75(8.24)$ & $70.25(10.771)$ & $66.92(6.45)$ & 0.067 \\
Weight $(\mathrm{kg})$ & $64.42(11.67)$ & $64.92(15.95)$ & $59.42(11.20)$ & 0.531 \\
Height (cm) & $160.67(11.67)$ & $162.75(10.01)$ & $164.92(7.38)$ & 0.577 \\
Onset period (mo) & $10.04(2.48)$ & $12.00(6.55)$ & $11.19(4.38)$ & 0.510 \\
Paralyzed side (right/left) & $5 / 7$ & $4 / 8$ & $5 / 7$ & 0.693 \\
MMSE-K & $28.04(1.85)$ & $28.15(1.88)$ & $26.50(2.17)$ & 0.743 \\
\hline
\end{tabular}

Values are presented as number only or mean (SD).

ADIM: abdominal draw-in maneuver, AEM: abdominal expansion maneuver, MMSE-K: Korean version of mini-mental status examination. 
Table 2. Comparison of the changes of center of pressure displacement in three groups

\begin{tabular}{|c|c|c|c|c|}
\hline & ADIM group $(n=12)$ & AEM group $(n=12)$ & Control group $(n=12)$ & $\mathrm{F}$ \\
\hline \multicolumn{5}{|c|}{ Medial-lateral-axis (cm) } \\
\hline Pre & $0.75(0.28)$ & $0.72(0.48)$ & $0.76(0.23)$ & 0.048 \\
\hline Post & $0.54(0.20)$ & $0.39(0.27)$ & $0.46(0.33)$ & 0.785 \\
\hline $\mathrm{t}$ & $2.816^{\mathrm{a}}$ & $2.926^{\mathrm{a}}$ & $2.460^{\mathrm{a}}$ & \\
\hline Change & $0.22(0.27)$ & $-0.33(0.63)$ & $-0.30(0.42)$ & 0.187 \\
\hline \multicolumn{5}{|c|}{ Anterior-posterior-axis $(\mathrm{cm})$} \\
\hline Pre & $0.61(0.17)$ & $0.52(0.26)$ & $0.51(0.08)$ & 0.915 \\
\hline Post & $0.33(0.22)$ & $0.36(0.28)$ & $0.37(0.15)$ & 1.032 \\
\hline $\mathrm{t}$ & $5.686^{\mathrm{a}}$ & $3.675^{\mathrm{a}}$ & $3.371^{\mathrm{a}}$ & \\
\hline Change & $-0.28(0.17)$ & $-0.17(0.35)$ & $-0.14(0.13)$ & 2.533 \\
\hline \multicolumn{5}{|c|}{ Sway mean velocity $(\mathrm{cm} / \mathrm{s})$} \\
\hline Pre & $2.59(0.51)$ & $2.77(0.36)$ & $2.61(0.43)$ & 0.615 \\
\hline Post & $2.34(0.47)$ & $2.13(0.65)$ & $2.42(0.37)$ & 1.015 \\
\hline $\mathrm{t}$ & $3.679^{\mathrm{a}}$ & $3.124^{\mathrm{a}}$ & $2.641^{\mathrm{a}}$ & \\
\hline Change & $-0.24(0.23)$ & $-0.64(0.71)^{b}$ & $-0.19(0.25)$ & $3.528^{\mathrm{a}}$ \\
\hline \multicolumn{5}{|c|}{ Sway area $95 \%\left(\mathrm{~cm}^{2}\right)$} \\
\hline Pre & $0.26(0.13)$ & $0.24(0.12)$ & $0.26(0.10)$ & 0.199 \\
\hline Post & $0.10(0.09)$ & $0.16(0.14)$ & $0.21(0.11)$ & 2.248 \\
\hline $\mathrm{t}$ & $5.908^{\mathrm{a}}$ & $3.539^{\mathrm{a}}$ & $2.615^{\mathrm{a}}$ & \\
\hline Change & $-0.15(0.09)^{b}$ & $-0.08(0.08)$ & $-0.06(0.07)$ & $5.001^{\mathrm{a}}$ \\
\hline
\end{tabular}

Values are presented as mean (SD).

ADIM: abdominal draw-in maneuver, AEM: abdominal expansion maneuver.

${ }^{\mathrm{a}} p<0.05$; ${ }^{\mathrm{b}}$ significantly different from control.

AEM experimental group and the control group $(p<0.05)$. In sway area at $95 \%$, a significant difference in all groups before and after the intervention $(p<0.05)$ was found. In Post-hoc test result, there was a difference in the ADIM experimental group and the control group $(p<0.05)$ (Table 2$)$.

\section{Discussion}

The purpose of this study was to investigate the effects of the trunk stabilization exercises such as ADIM and AEM in upright undisturbed stance with unsupported sitting position in stroke patients. In addition, we analyzed the change of COP displacement in the sitting posture of the stroke patients according to trunk stabilization exercises in biomechanics.

For the AEM training conducted in this study, the diaphragm, pelvic lower muscles and the rectus abdominis regulate the IAP and provide the stability required for pelvic posture. Thus inherent spinal stabilization muscles with IAP cooperatively provide spine rigidity, which serves to provide dynamic stability of the spine. This method was used to push the abdomen outward with the abdominal breathing method using the diaphragm to increase the IAP during trunk stabilization exercises [22]. This does not extend to the chest during inspiration while the lower abdominal expands. If the navel extends in the caudal anterior direction, it may have the advantage of being able to increase the IAP, the simultaneous contraction of the diaphragm, and deep spinal stabilizer muscles. ADIM training was performed in the prone position using a pressure bio-feedback system, which has been commonly used to give stability to the pelvis and lower back area during movement of the upper and lower limbs [14]. We performed the ADIM training in the prone position with adults with instability that was reported of the thickened core muscle, the transverse abdominis, and obliqus abdominus interni. Another previous study had also reported that the ADIM increases the activity of transverse abdominis and obliqus abdominus interni of the deep abdominal muscles [24].

In a sitting position, an upright stance is controlled through a variety of senses of visual, vestibular, and proprioceptive sensory and tactile plantar input. In an undisturbed sitting position in order to maintain posture, sensory and mechanical changes are important [25]. In the level of sensory, tactile plantar of both lower extremities and threshold of somatosensory have less participation to maintain the sitting 
position, therefore the tactile sensation of the hips is used. The centre of gravity (COG) is lowered and the base of support is widened, which decreases the number of joints to be controlled at the level of movement. The sitting position is maintained by controlling the pelvic joint using the trunk muscles. Despite these differences, the control ability related to the sitting position is seen with the same characteristics as standing [26]. However, compared to the standing position, in order to maintain the sitting position by biomechanical limit is reduced, the lower the size of the COP movement [26]. This phenomenon is a result of the reduced co-contraction of agonist and antagonist in the stability of the sitting position.

We analyzed the changes of the COP displacement in the sitting posture using the WBB. It can assess the patient objectively. Also, we can use the COP data of correct parameter to express the sitting position for rehabilitation protocol. It is also possible to take advantage of objective indicators than clinical evaluation in relation to the postural stability of the stroke patients through it [21]. Therefore, biomechanical evaluation is also important as clinical evaluation provides a wealth of information about the undisturbed sitting posture in stroke patients. COP trajectory is the phenomenon of muscle activity which appears to control the physical stability, with the movement of the $\mathrm{COG}$, it is possible to quantitatively evaluate the COP displacement of body sway. When sitting position of stroke patients is compared to normal subjects, the increase in range and average speed of the COP are consistent with the results of the clinical evaluation [20]. Trunk control test, postural assessment scale for stroke patients or Fugl-Meyer results of the evaluation of stroke patients had lower scores on some items showing trunk function disorder (flexion/extension; adduction/abduction, transfer), and the ability to maintain posture (dynamic and undisturbed) was lowered [17,27]. This phenomenon is caused by the wrong perception of the body schema and the reduced strength of the trunk muscles of the stroke patients [28].

The ML and AP axes to the movement were caused by differences in control of anterior, posterior, and lateral trunk muscles movement of the COP in a sitting position. In a previous study of the recovery period of 20 days after stroke onset, the recovery of AP axis of COP movement was developing faster than the recovery of ML axis. These results can be seen when sagittal equilibrium is damaged greater than the frontal equilibrium [25]. Mostly, damage to the left and right side of the obliqus abdominus externi and obliqus abdominus interni that is responsible for the movement of the trunk side indicates that damage of both sides are antagonistic of the rectus abdominis and latissimus dorsi muscles to control trunk flexion and extension is greater [29]. In this study, no significant differences were found in the comparison of ADIM experimental group, AEM experimental group, and control group in the changes of the ML and AP axes. This is the result due to improved low range of the chronic stroke patients used in this study compared to acute or subacute stroke patients. In the sitting posture of stroke patients, COP velocity represents the posture stability, and an increase in the velocity represents a decrease of the posture stability. Furthermore, the increase in the sway area increases the risk of falling [30]. Thus in the trunk stabilization exercises performed in this study, the AEM experimental group showed greater improvement in the COP velocity items, and the ADIM experimental group showed greater improvement in the sway area items when comparing the two groups of the different variables. This is expected to have a positive effect on the fall prevention and increased sitting position stability.

This study was performed to present a more effective method intervention and improvement of safe upright undisturbed posture in an unsupported sitting position. This research is difficult to generalize for all stroke patients because we used subjects with chronic stroke, the number of subjects was small, and in the case of AEM training, feedback and quantification is difficult it has the limitation that require continuous observation of the therapist during intervention. In future studies, it will be necessary to study trunk stabilization training in acute stroke patients.

After trunk stabilization training for stroke patients, the change of sway area of the ADIM experimental group, and the change of sway velocity of the AEM experimental group showed significant improvements than in the control group. It can be concluded that both AEM and ADIM training is an necessary intervention to maintain the independent sitting position according to the characteristics of the patient, and it is believed to have a positive impact on the initial standing balance and the ability of gait improvement of stroke patients.

\section{Conflict of Interest}

The authors declared no potential conflicts of interest with respect to the authorship and/or publication of this article. 


\section{References}

1. Jandt SR, Caballero RM, Junior LA, Dias AS. Correlation between trunk control, respiratory muscle strength and spirometry in patients with stroke: an observational study. Physiother Res Int 2011;16:218-24.

2. Kolb B, Gibb R. Brain plasticity and recovery from early cortical injury. Dev Psychobiol 2007;49:107-18.

3. Davies PM, Klein-Vogelbach S. Right in the middle: selective trunk activity in the treatment of adult hemiplegia. Berlin: Springer Science \& Business Media; 1990.

4. Wang CH, Hsueh IP, Sheu CF, Hsieh CL. Discriminative, predictive, and evaluative properties of a trunk control measure in patients with stroke. Phys Ther 2005;85:887-94.

5. Dietz V. Human neuronal control of automatic functional movements: interaction between central programs and afferent input. Physiol Rev 1992;72:33-69.

6. Skinner JS. Exercise testing and exercise prescription for special cases: theoretical basis and clinical application. Philadelphia: Lippincott Williams \& Wilkins; 2005.

7. Howard RS, Rudd AG, Wolfe CD, Williams AJ. Pathophysiological and clinical aspects of breathing after stroke. Postgrad Med J 2001;77:700-2.

8. Teixeira-Salmela LF, Parreira VF, Britto RR, Brant TC, Inácio EP, Alcântara TO, et al. Respiratory pressures and thoracoabdominal motion in community-dwelling chronic stroke survivors. Arch Phys Med Rehabil 2005;86:1974-8.

9. Hodges PW, Richardson CA. Inefficient muscular stabilization of the lumbar spine associated with low back pain. A motor control evaluation of transversus abdominis. Spine (Phila Pa 1976) 1996;21:2640-50.

10. van de Port IG, Kwakkel G, Schepers VP, Lindeman E. Predicting mobility outcome one year after stroke: a prospective cohort study. J Rehabil Med 2006;38:218-23.

11. Geurts AC, de Haart M, van Nes IJ, Duysens J. A review of standing balance recovery from stroke. Gait Posture 2005;22:267-81.

12. Verheyden G, Nieuwboer A, De Wit L, Feys H, Schuback B, Baert I, et al. Trunk performance after stroke: an eye catching predictor of functional outcome. J Neurol Neurosurg Psychiatry 2007;78:694-8.

13. Kolar P. Clinical rehabilitation. Praha: Alena Kobesová; 2014.

14. Yoon MR, Choi HS, Shin WS. Effects of the abdominal drawing-in maneuver and the abdominal expansion maneuver on grip strength, balance and pulmonary function in stroke patients. $\mathrm{J}$ Kor Phys Ther 2015;27:147-53.

15. Macedo LG, Maher CG, Latimer J, McAuley JH. Motor control exercise for persistent, nonspecific low back pain: a systematic review. Phys Ther 2009;89:9-25.
16. Teyhen DS, Miltenberger CE, Deiters HM, Del Toro YM, Pulliam JN, Childs JD, et al. The use of ultrasound imaging of the abdominal drawing-in maneuver in subjects with low back pain. J Orthop Sports Phys Ther 2005;35:346-55.

17. Franchignoni FP, Tesio L, Ricupero C, Martino MT. Trunk control test as an early predictor of stroke rehabilitation outcome. Stroke 1997;28:1382-5.

18. Verheyden G, Nieuwboer A, Mertin J, Preger R, Kiekens C, De Weerdt W. The Trunk Impairment Scale: a new tool to measure motor impairment of the trunk after stroke. Clin Rehabil 2004; 18:326-34.

19. Benaim C, Pérennou DA, Villy J, Rousseaux M, Pelissier JY. Validation of a standardized assessment of postural control in stroke patients: the Postural Assessment Scale for Stroke Patients (PASS). Stroke 1999;30:1862-8.

20. van Nes IJ, Nienhuis B, Latour H, Geurts AC. Posturographic assessment of sitting balance recovery in the subacute phase of stroke. Gait Posture 2008;28:507-12.

21. Perlmutter S, Lin F, Makhsous M. Quantitative analysis of static sitting posture in chronic stroke. Gait Posture 2010;32:53-6.

22. Frank C, Kobesova A, Kolar P. Dynamic neuromuscular stabilization \& sports rehabilitation. Int J Sports Phys Ther 2013;8:62-73.

23. Park DS, Lee DY, Choi SJ, Shin WS. Reliability and validity of the balancia using wii balance board for assessment of balance with stroke patients. J Korea Acad Industr Coop Soc 2013;14: 2767-72.

24. Yun JH, Kim TS, Lee BK. The effects of combined complex exercise with abdominal drawing-in maneuver on expiratory abdominal muscles activation and forced pulmonary function for post stroke patients. J Korean Soc Phys Med 2013;8:513-23.

25. Genthon N, Vuillerme N, Monnet JP, Petit C, Rougier P. Biomechanical assessment of the sitting posture maintenance in patients with stroke. Clin Biomech (Bristol, Avon) 2007;22:1024-9.

26. Genthon N, Rougier P. Does the capacity to appropriately stabilize trunk movements facilitate the control of upright standing? Motor Control 2006;10:232-43.

27. Hsieh CL, Sheu CF, Hsueh IP, Wang CH. Trunk control as an early predictor of comprehensive activities of daily living function in stroke patients. Stroke 2002;33:2626-30.

28. Bohannon RW, Smith MB, Larkin PA. Relationship between independent sitting balance and side of hemiparesis. Phys Ther 1986;66:944-5

29. Zedka M, Kumar S, Narayan Y. Electromyographic response of the trunk muscles to postural perturbation in sitting subjects. $\mathbf{J}$ Electromyogr Kinesiol 1998;8:3-10.

30. Nichols DS, Miller L, Colby LA, Pease WS. Sitting balance: its relation to function in individuals with hemiparesis. Arch Phys Med Rehabil 1996;77:865-9. 